\title{
Greater Trochanteric Pain Syndrome
}

\author{
Andrea S. Klauser, MD ${ }^{1}$ Carlo Martinoli, MD ${ }^{2}$ Alberto Tagliafico, MD ${ }^{3}$ Rosa Bellmann-Weiler, MD \\ Gudrun M. Feuchtner, MD, PhD ${ }^{1}$ Marius Wick, MD ${ }^{1}$ Werner R. Jaschke, MD, PhD ${ }^{1}$
}

${ }^{1}$ Department of Diagnostic Radiology, Innsbruck Medical University,
Innsbruck, Austria
${ }^{2}$ Cattedra “R" di Radiologia-DICMI, University of Genoa, Genoa, Italy
${ }^{3}$ Department of Experimental Medicine-DIMES, Institute of Anatomy,
University of Genoa, Genoa, Italy
${ }^{4}$ Department of Internal Medicine I, Clinical Immunology and
Infectious Diseases, Innsbruck Medical University, Innsbruck, Austria

Address for correspondence Andrea S. Klauser, MD, Department of Radiology, Innsbruck Medical University, Anichstrasse 35, A-6020 Innsbruck, Austria (e-mail: andrea.klauser@i-med.ac.at).

Semin Musculoskelet Radiol 2013;17:43-48.

\begin{abstract}
Keywords

- gluteus

- greater trochanter

- ultrasound

- bursa

Pain around the greater trochanter is still a common clinical problem that may be secondary to a variety of either intra-articular or periarticular pathologies. Gluteal tendon pathologies are one of the primary causes of greater trochanteric pain, with attrition of the fasciae latae against the gluteus medius and minimus tendons, and the trochanteric bursa being possible causes. Key sonographic findings of gluteal tendinopathy, bursitis, and differential diagnosis are described in this overview. Clinical diagnosis and treatment of greater trochanteric pain syndrome is still challenging; therefore ultrasound is helpful to localize the origin of pain, determine underlying pathology, and, based on these findings, to guide local aspiration and/or injection in cases of tendinopathy and/or bursitis.
\end{abstract}

Pain around the greater trochanter is still a common clinical problem that may be secondary to a variety of either intraarticular or periarticular pathologies that include trauma, osteonecrosis, infection, stress fractures of the femoral neck, radiated pain from the spine, entrapment neuropathies, trochanteric bursitis, and tendinopathies of the gluteus medius and minimus muscles. However, gluteal tendon pathologies are one of the most common pathologies causing lateral hip pain. Ultrasound (US) is able to distinguish gluteus tendinopathy from trochanteric bursitis, assess snapping hip related to the iliotibial band, and differentiate MorelLavallée lesions from other masses. ${ }^{1,2}$ However, the primary indications for a US examination of the lateral hip are tendinopathy and bursitis around the trochanteric region. ${ }^{3-5}$

The pathogenesis of greater trochanteric pain syndrome is not completely understood, although rheumatologists and orthopedic surgeons are becoming increasingly aware that local microtrauma leading to tears of the abductor tendons may be a leading cause of this syndrome., ${ }^{6,7}$ From the pathophysiologic point of view, some analogies between gluteal tendinopathy and shoulder impingement can be hypothe- sized, with attrition of the fasciae latae against the tendons of the gluteus medius and minimus and the trochanteric bursa, similar to that of the acromion with the rotator cuff and the subacromial-subdeltoid bursa. ${ }^{8}$

Symptoms are typically located unilaterally, although degenerative findings may often be seen bilaterally. Gluteal tendinopathy most frequently occurs in late middle-aged women. The range of hip motion is not affected, but the patient reports pain when lying on the affected side or climbing stairs. Standard radiographs may reveal either intratendinous or bursal calcifications, or enthesopathy, although pathologic changes are not detectable in most patients. Because underlying pathologies cannot be distinguished on the basis of clinical examination and findings, they are conventionally grouped in the greater trochanteric pain syndrome. ${ }^{9}$ US has the potential to delineate the conditions in greater trochanteric pain syndrome described in this review.

\section{Tendinopathy}

Greater trochanteric pain syndrome is commonly due to gluteus minimus and medius overuse or injury rather than
Issue Theme Musculoskeletal Ultrasound Update; Guest Editor, Jon A. Jacobson, MD.
Copyright @ 2013 by Thieme Medical Publishers, Inc., 333 Seventh Avenue, New York, NY 10001, USA. Tel: +1(212) 584-4662.
DOI http://dx.doi.org/ 10.1055/s-0033-1333913. ISSN 1089-7860. 


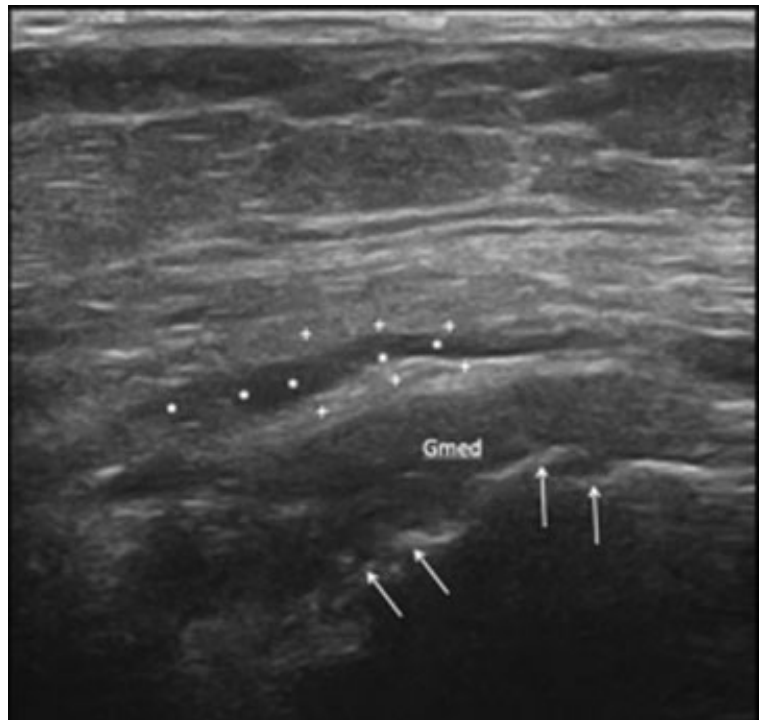

Fig. 1 Gluteus medius (Gmed) tendinopathy and bursal fluid. US image longitudinal to gluteus medius shows heterogeneous echogenicity with diffuse tendon thickening at the medial gluteal tendon insertion. Arrows shows cortical irregularity of the greater trochanter at the tendon insertion. Stars show effusion in the trochanteric bursa with chronic induration of the bursal layers as well (crosses in echogenic surrounding of bursa).

trochanteric bursitis only. Patients typically complain of a pinpoint pain over the lateral and posterior aspect of the greater trochanter associated with tenderness at deep pressure. Because of this predominant finding, sonopalpation is extremely helpful in localization of the pain origin and for further therapeutic managements such as sonographic-guided injections for these conditions.

The concept of the four greater trochanter facets is well demonstrated by US aiding in correct identification of the affected tendons. ${ }^{10,11}$ The gluteus minimus and gluteus medius tendons attach onto the anterior and lateral facets, respectively. This appearance has been likened to the rotator cuff of the shoulder. ${ }^{8}$ The gluteus minimus insertion is variable and ranges from a triangular to an irregular $\mathrm{L}$ shape at the anterior facet. The anterior gluteus medius tendon has a

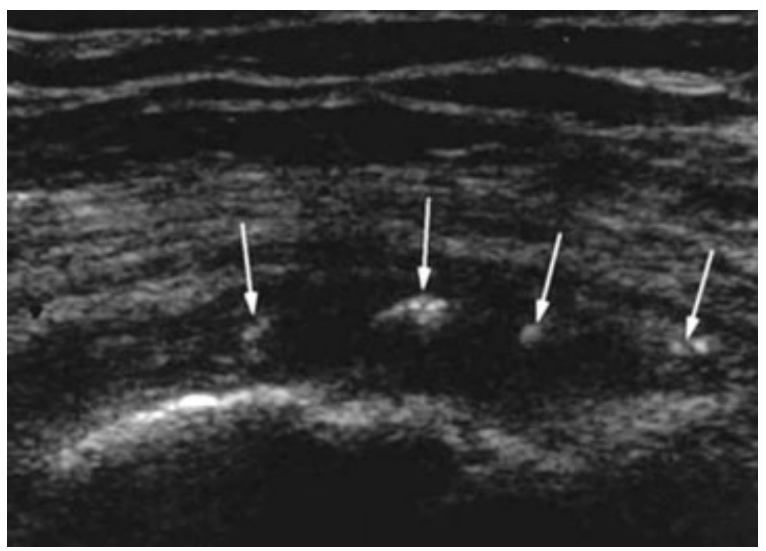

Fig. 2 Calcific tendinosis. US image longitudinal to the gluteus medius tendon shows intratendinous echogenic foci (arrows). broad attachment on the lateral facet, whereas the posterior part has also a narrow attachment on the superolateral facet. Most patients exhibit abnormalities in the anterior and posterior portion of the gluteus medius; an effusion inside the trochanteric bursa can be an associated finding ( $\boldsymbol{- F i g . ~ 1 ) . ~}$

US signs of gluteal tendinopathy are based on the development of different tendinotic stages. Tendinosis is defined by a loss of fibrillar echotexture, loss of echogenicity due to edema and mucoid degeneration, heterogeneous echogenicity, altogether often present with tendon thickening that can be diffuse or focal. The tendon of the gluteus minimus or the anterior and posterior tendons of the gluteus medius can be involved alone or in association. However, the anterior portion of the gluteus medius is the most commonly affected; only a minority of patients show changes in the gluteus minimus. ${ }^{6}$ Increased vascularity on color Doppler is an uncommon finding. ${ }^{12}$ The affected tendon may be tender to sonopalpation.

Calcific tendinosis within the gluteal tendons or at the insertion in terms of enthesopathy can be further present and is better seen on US than MRI by showing hyperechoic welldefined or ill-defined echogenic depositions (-Fig. 2). US can be used to guide aspiration to treat these calcifications.

\section{Tendon Tears}

Tendon tears are manifested by tendon thinning and partialor full-thickness anechoic defects within the tendon (-Fig. 3). Partial-thickness tears typically involve the deep portion of the anterior gluteus medius tendon. ${ }^{6}$ Ruptures can also be found at the insertion and in the tendon substance itself. In full-thickness tears, the tendon appears retracted proximally with anechoic effusion related to the hematoma seen between it and the naked greater trochanter filling the trochanteric bursa. A "bold" facet with absence of tendon fibers is compatible with a complete tear ( -Figs. 4-6). Muscle wasting

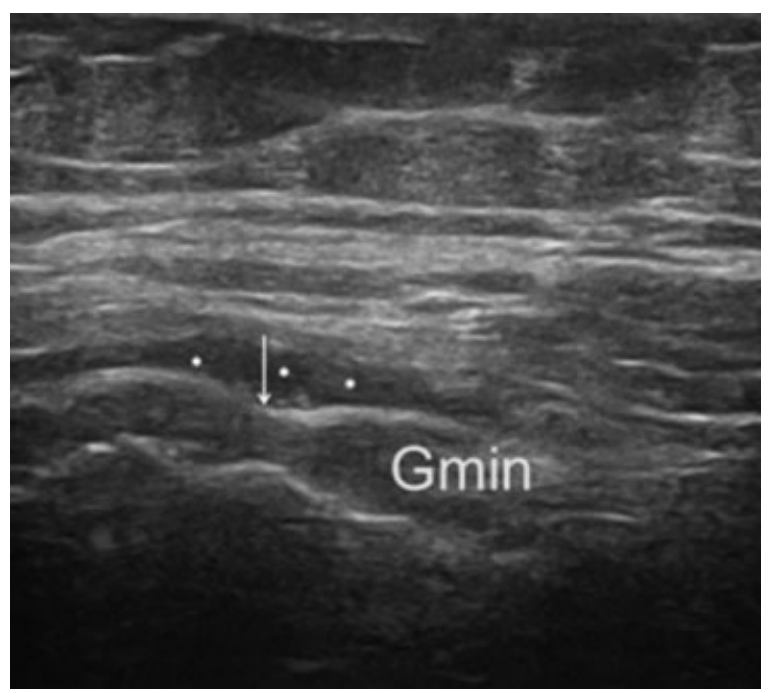

Fig. 3 Gluteus minimus: partial-thickness tear. US image longitudinal to the gluteus minimus ( $\mathrm{Gmin}$ ) shows partial-thickness tear of the Gmin tendon, which appears as loss of convexity (arrow) and herniation of overlying bursitis (stars). 


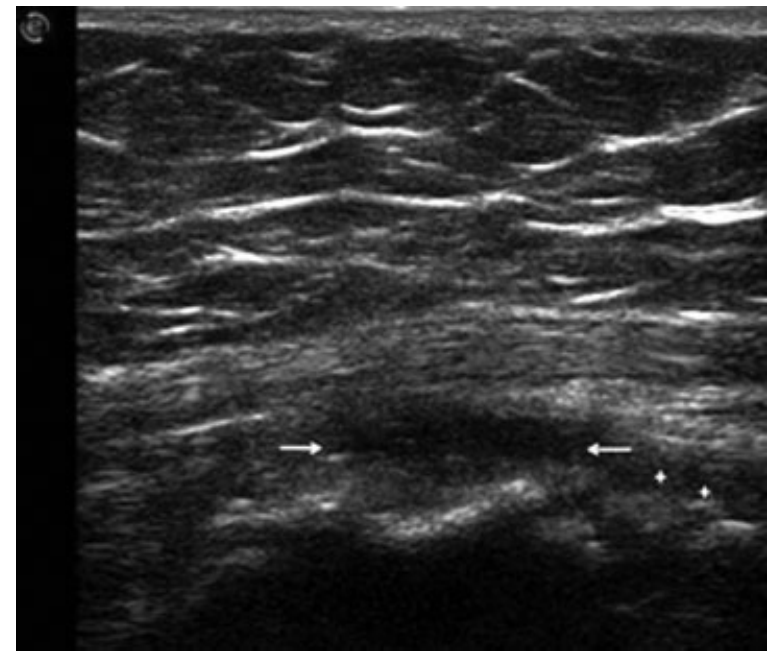

Fig. 4 Gluteus medius: complete tear. US image transverse to the anterior gluteus medius tendon shows complete rupture (between arrows). Note gluteus minimus tendon (crosses).

may also be evident with loss of muscle bulk and increased echogenicity due to fat replacement (-Fig. 7).In the presence of cortical irregularities deep to the gluteal insertions, a careful evaluation of the overlying tendon, as in the rotator cuff of the shoulder, should be performed to visualize a potential partial-thickness tear ( $\mathbf{- F i g . 1}$ ).

\section{Bursitis}

Histopathologic findings have shown tendinopathy and bursa pathology coexisting in greater trochanteric pain syndrome. ${ }^{13}$ It has been shown that bursal distention is not typically inflamed and often is not the primary cause of symptoms. ${ }^{14}$ To better localize all bursae, their anatomical location is as follows: The subgluteus minimus bursa is located between the gluteus minimus tendon and the anteri- or facet. The subgluteus medius bursa predominantly lies immediately deep to the portion of the lateral insertion of the gluteus medius tendon. The trochanteric bursa (or subgluteus maximus bursa) covers the posterior facet, lying deep to the gluteus maximus muscle extending laterally and distally. ${ }^{11} \mathrm{~A}$ cadaveric study by Dunn et al showed a variable anatomy of the trochanteric bursa ranging from the absence to the presence of multiple bursae in different specimens with increasing prevalence with older age. Additional bursae may be seen between the iliotibial band and the greater trochanter ${ }^{10}$ ( $\mathbf{- F i g s . ~} 8$ and $\mathbf{9}$ ).

Acute bursal fluid collections are readily appreciated as a hypoechoic thin fluid accumulation. Fluid distension of the trochanteric bursa appears as a well-circumscribed crescentshaped hypoechoic to anechoic collection located at the lateral aspect of the greater trochanter deep to the gluteus maximus, which can extend superficially over the posterior insertion of the gluteus medius (-Fig. 10).

Chronic bursitis, which is commonly present in chronic tendon disease, can show effusions as well; however, isoechoic bursal wall thickening can be present that might be easily overlooked. Symptoms in these conditions might be prevalent due to adhesions of the bursal layers.

It is not always possible to differentiate trochanteric bursitis from an underlying tendinopathy, and, in many instances, the two conditions can coexist (-Fig. 9).

Trochanteric bursitis can be interpreted as a true impingement syndrome ${ }^{15}$ from the pathophysiologic point of view. In addition, in hip abductor tendons tear, impingement of the greater trochanter on the fasciae latae may develop, thus sustaining chronic bursitis. Again, especially in these cases, sonopalpation is of great diagnostic value.

\section{Scanning and Injection Technique}

Patients are positioned on their contralateral side for better access to the area of lateral hip pain. ${ }^{16,17}$ The symptomatic hip

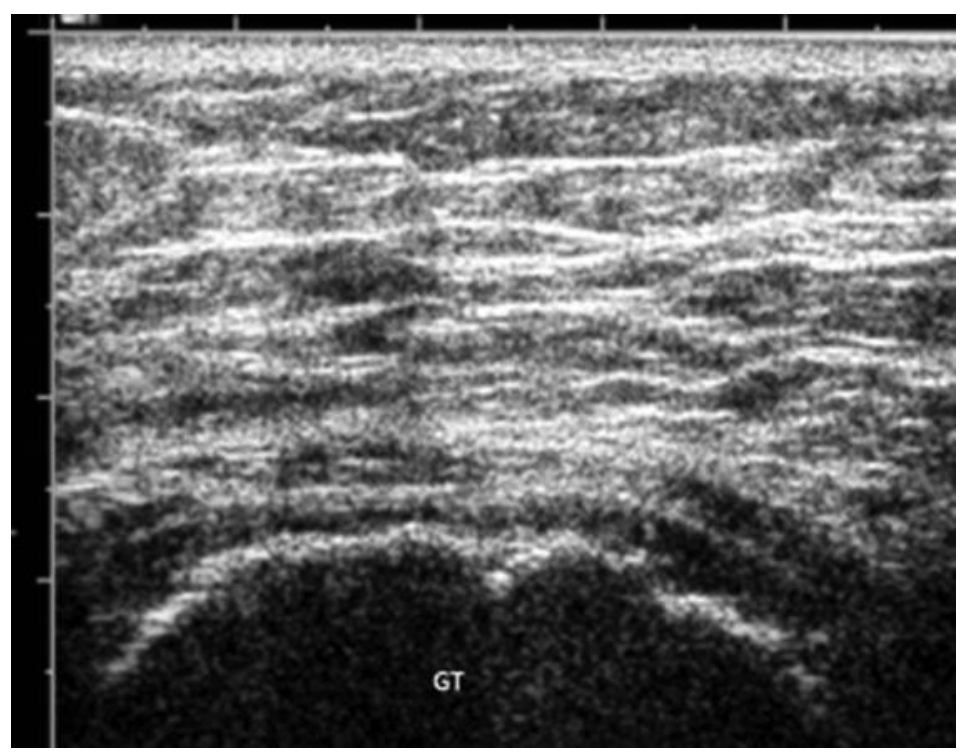

Fig. 5 Gluteus medius and minimus: complete tear. US image short axis to gluteal tendons shows naked trochanter (GT) with complete rupture of the gluteus medius and minimus tendon. 

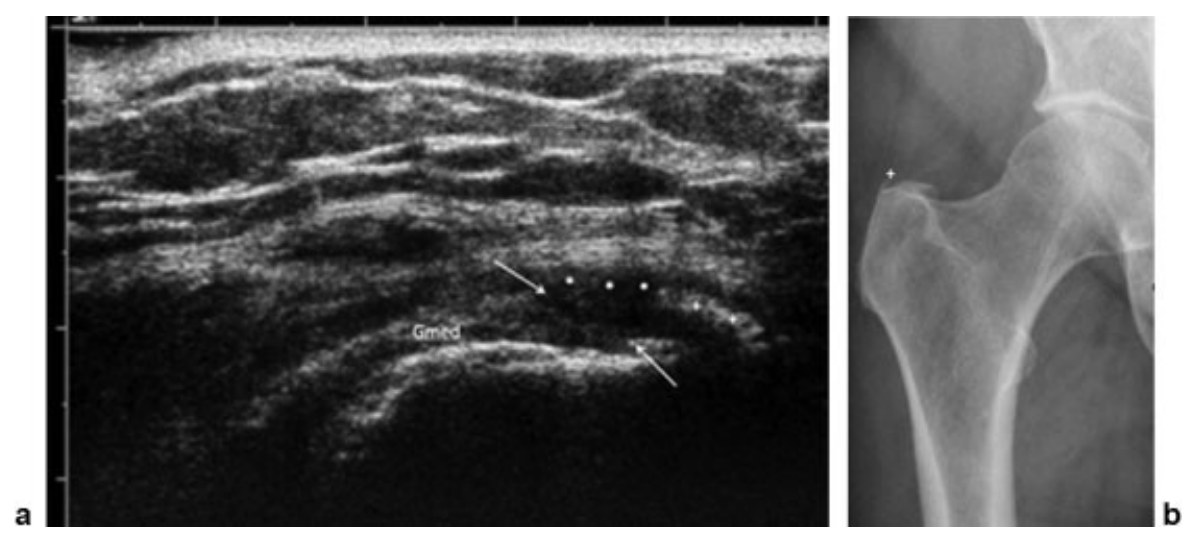

Fig. 6 Gluteus medius tendon: full-thickness tear. (a) US image shows transmural rupture of the anterior gluteus medius (Gmed) tendon (between arrows), overlying fluid (stars), and bony spur ventrally (crosses). (b) Corresponding radiograph shows the bony spur (cross) at the greater trochanter.

is slightly flexed. First a longitudinal parasagittal scanning plane is acquired, where bursitis can be better delineated; then an axial investigation is added by including sonopalpation for pain localization.

US-guided injections are performed with a 25-gauge needle via a transverse or longitudinal approach using a mixture of corticosteroids and long-acting local anesthetics (-Fig. 11).

The first treatment of gluteus tendinopathy includes rest and physical therapy, but often local steroid injections are only useful in refractory cases.

US guidance can increase the efficacy of local therapy by allowing accurate insertion of the medication inside the affected bursa or near the degenerated tendon. ${ }^{18}$ Furthermore, bursal effusion should be aspirated to improve medication

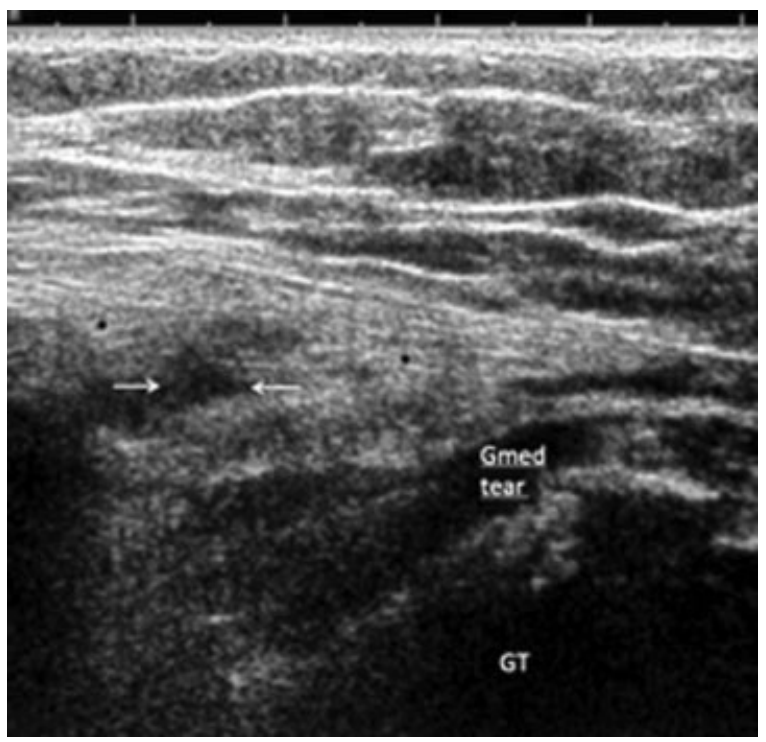

Fig. 7 Gluteus medius: full-thickness tear and muscle atrophy. US image transverse to the gluteus medius at the dorsal aspect of the greater trochanter (GT) shows complete tear of the gluteus medius tendon (Gmed tear) with muscle wasting, fluid extending to the musculotendinous junction (between arrows), and increased muscle echogenicity.

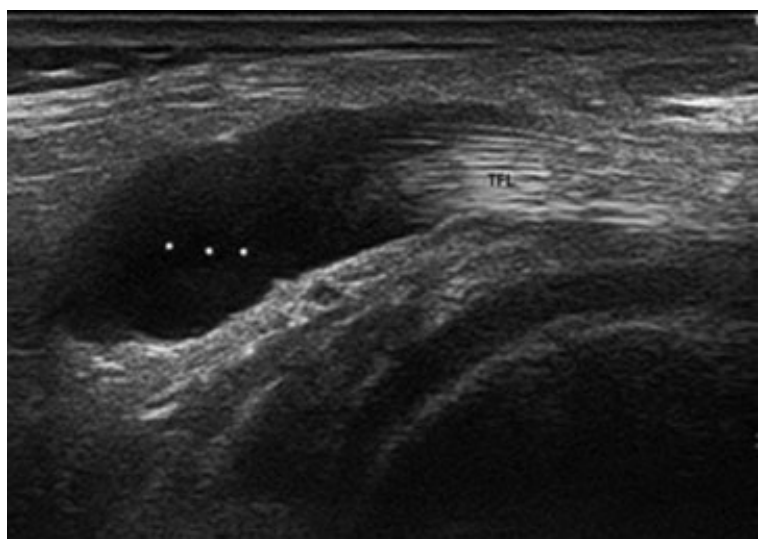

Fig. 8 Trochanteric bursal effusion. US image over the greater trochanter shows effusion (stars) in the trochanteric bursa under the tensor fasciae latae (TFL) tendon.US can guide aspiration to evacuate the fluid, followed by a corticosteroid injection.

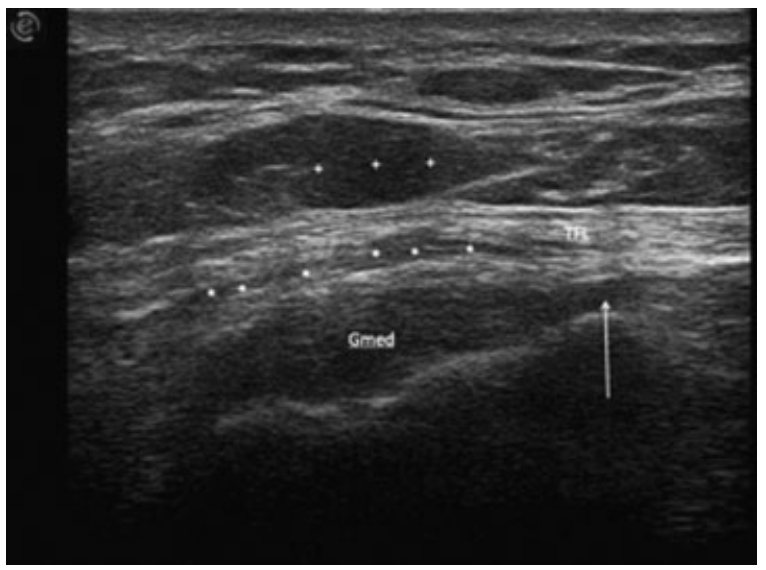

Fig. 9 Gluteus medius tendinosis, bursal effusion, and Morel-Lavallée lesion. US image long axis to gluteus medius (Gmed) shows tendinosis of the gluteus medius insertion with loss of echogenicity due to edema and mucoid degeneration. Distally, gluteus medius thinning (arrow) indicates chronic partial rupture. There is also an effusion in the trochanteric bursa (stars) with chronic induration of the bursal layers (echogenic periphery). Note Morel-Lavallée lesion (crosses) lying superficially to the tensor fasciae latae (TFL) tendon, an important differential diagnosis easily visualized by US. 


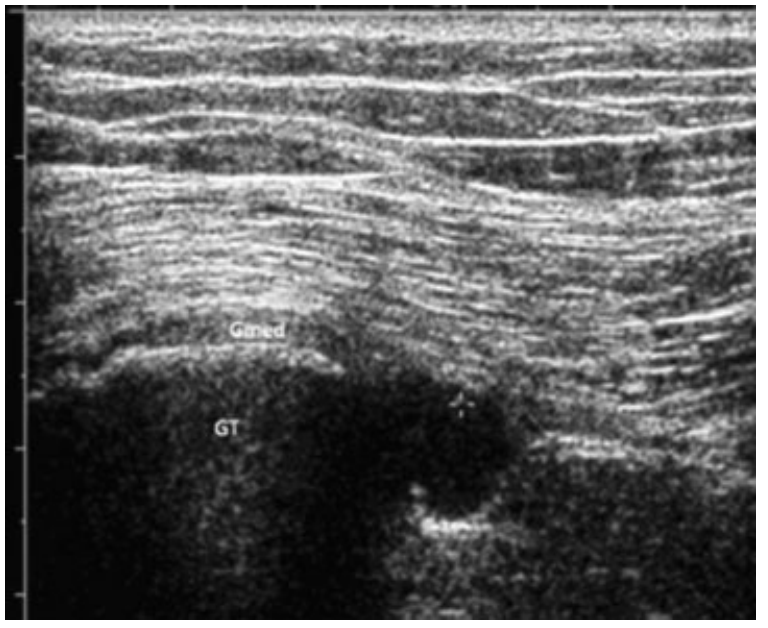

Fig. 10 Axial scan from dorsal at the greater trochanter (GT): deepseated effusion (cross) in the trochanteric bursa. Thinning of the dorsal part of gluteus medius tendon (Gmed).

concentration and reduce any possible impingement condition. For this purpose, the needle should be guided inside the affected bursa or bursae by approaching the greater trochanter from a lateral or posterolateral direction, depending on the localization of the affected bursa. ${ }^{19-22}$ US-guided tendon fenestration may also be a consideration. ${ }^{23}$

The European Society of Skeletal Radiology Ultrasound Group summarized the value of US in terms of evidence in trochanteric pain as grade C evidence only; however, expert consensus rated the overall value of US because of the potential for injections as "2," defined as equivalent to other imaging techniques (other techniques might provide significant information). ${ }^{3}$ In avulsion injuries, surgery with debridement and tendon reattachment might be indicated.

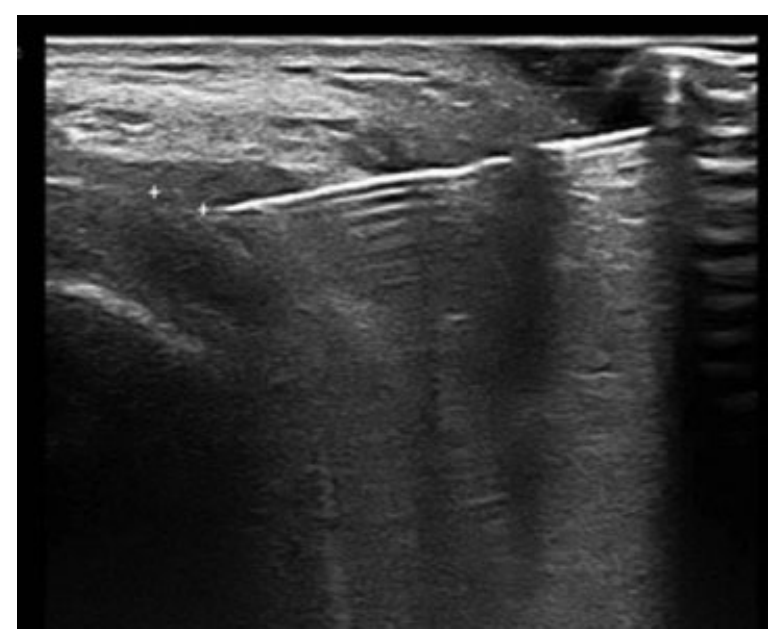

Fig. 11 US-guided injection of the subgluteus medius bursa (crosses) showing the exact needle location.

\section{Summary}

Many conditions are associated with greater trochanteric pain syndrome, such as gluteal tendinopathy and bursitis, which are well demonstrated by US. Sonopalpation is further helpful, and US-guided injections based on sonopathologic findings can provide diagnostic information and guide treatment for this often unclear clinical condition.

\section{References}

1 Neal C, Jacobson JA, Brandon C, Kalume-Brigido M, Morag Y, Girish G. Sonography of Morel-Lavallee lesions. J Ultrasound Med 2008;27(7):1077-1081

2 Bass CJ, Connell DA. Sonographic findings of tensor fascia lata tendinopathy: another cause of anterior groin pain. Skeletal Radiol 2002;31(3):143-148

3 Klauser AS, Tagliafico A, Allen GM, et al. Clinical indications for musculoskeletal ultrasound: a Delphi-based consensus paper of the European Society of Musculoskeletal Radiology. Eur Radiol 2012;22(5):1140-1148

4 Bancroft LW, Merinbaum DJ, Zaleski CG, Peterson JJ, Kransdorf MJ, Berquist TH. Hip ultrasound. Semin Musculoskelet Radiol 2007;11 (2):126-136

5 Martinoli C, Garello I, Marchetti A, et al. Hip ultrasound. Eur J Radiol 2012;81(12):3824-3831

6 Connell DA, Bass C, Sykes CA, Young D, Edwards E. Sonographic evaluation of gluteus medius and minimus tendinopathy. Eur Radiol 2003;13(6):1339-1347

7 Cvitanic O, Henzie G, Skezas N, Lyons J, Minter J. MRI diagnosis of tears of the hip abductor tendons (gluteus medius and gluteus minimus). AJR Am J Roentgenol 2004;182(1):137-143

8 Bunker TD, Esler CN, Leach WJ. Rotator-cuff tear of the hip. J Bone Joint Surg Br 1997;79(4):618-620

9 Karpinski MR, Piggott H. Greater trochanteric pain syndrome. A report of 15 cases. J Bone Joint Surg Br 1985;67(5): 762-763

10 Dunn T, Heller CA, McCarthy SW, Dos Remedios C. Anatomical study of the "trochanteric bursa." Clin Anat 2003;16(3): 233-240

11 Pfirrmann CW, Chung CB, Theumann NH, Trudell DJ, Resnick D. Greater trochanter of the hip: attachment of the abductor mechanism and a complex of three bursae-MR imaging and MR bursography in cadavers and MR imaging in asymptomatic volunteers. Radiology 2001;221(2):469-477

12 Kong A, Van der Vliet A, Zadow S. MRI and US of gluteal tendinopathy in greater trochanteric pain syndrome. Eur Radiol 2007;17 (7):1772-1783

13 Fearon AM, Scarvell JM, Cook JL, Smith PN. Does ultrasound correlate with surgical or histologic findings in greater trochanteric pain syndrome? A pilot study. Clin Orthop Relat Res 2010;468 (7):1838-1844

14 Silva F, Adams T, Feinstein J, Arroyo RA. Trochanteric bursitis: refuting the myth of inflammation. J Clin Rheumatol 2008;14(2): $82-86$

15 Heller A. Anatomy of the trochanteric bursae [letter]. Radiology 2003;226(3):921; author reply 921-922

16 Bianchi S, Martinoli C. Lateral hip pain. In Bianchi S, Martinoli C, eds. Ultrasound of the Musculoskeletal System. Berlin, Germany: Springer Verlag; 2007:589-594

17 Beggs I, Bianchi S, Bueno A, et al. Musculoskeletal ultrasound technical guidelines. IV. Hip. European Society of Musculoskeletal Radiology. Available at: http://www.essr.org/html/img/pool/hip. pdf. Accessed August 10, 2011 
18 Labrosse JM, Cardinal E, Leduc BE, et al. Effectiveness of ultrasound-guided corticosteroid injection for the treatment of gluteus medius tendinopathy. AJR Am J Roentgenol 2010;194(1):202-206

19 Rowbotham EL, Grainger AJ. Ultrasound-guided intervention around the hip joint. AJR Am J Roentgenol 2011;197(1):W122-W127

20 Adler RS, Sofka CM. Percutaneous ultrasound-guided injections in the musculoskeletal system. Ultrasound Q 2003;19(1):3-12
21 De ZordoT, Mur E, Bellmann-Weiler R, et al. US guided injections in arthritis. Eur J Radiol 2009;71(2):197-203

22 Sofka CM, Saboeiro G, Adler RS. Ultrasound-guided adult hip injections. J Vasc Interv Radiol 2005;16(8):1121-1123

23 Housner JA, Jacobson JA, Misko R. Sonographically guided percutaneous needle tenotomy for the treatment of chronic tendinosis. J Ultrasound Med 2009;28(9):1187-1192 